\title{
ELLIPTIC QUANTUM GROUPS AND RUIJSENAARS MODELS
}

\author{
GIOVANNI FELDER* AND ALEXANDER VARCHENKO**,1 \\ *D-MATH, ETH-Zentrum, \\ 8092 Zürich, Switzerland \\ ** Department of Mathematics, University of North Carolina at Chapel Hill, \\ Chapel Hill, NC 27599-3250, USA
}

March 1997

\begin{abstract}
We construct symmetric and exterior powers of the vector representation of the elliptic quantum groups $E_{\tau, \eta}\left(g l_{N}\right)$. The corresponding transfer matrices give rise to various integrable difference equations which could be solved in principle by the nested Bethe ansatz method. In special cases we recover the Ruijsenaars systems of commuting difference operators.
\end{abstract}

\section{INTRODUCTION}

Elliptic quantum groups [E] are the algebraic structure underlying quantum integrable models of statistical mechanics involving elliptic functions, and the $q$-deformation of conformal field theory on elliptic curves.

The basic object, appearing in the presentation of elliptic quantum groups by quadratic relations, is a "dynamical $R$-matrix", a solution of a modification of the YangBaxter equation. This $R$-matrix depends on the spectral parameter and on an additional parameter lying in the Cartan subalgebra of a simple Lie algebra. The usual YangBaxter equation is obtained in the limit when the latter parameter tends to infinity. However, for elliptic solutions, a limit exists only in trigonometric degenerations.

In this paper we start a study of the representation theory of elliptic quantum groups of type $A_{N-1}$, and give some applications. We construct, using the fusion procedure KRS, Q, JKMO, analogues of the symmetric and exterior powers of the vector representation of $g l_{N}$. Out of these representations, and their tensor products, one can construct new dynamical $R$-matrices. Taking partial traces of these $R$-matrices gives rise to families of commuting difference operators. In a special case, we recover the Ruijsenaars system $[\mathbb{R}]$ of difference operators, which is the difference (or "relativistic") analogue of the Calogero-Moser integrable system of differential operators. In particular, we obtain a simpler proof of the commutativity of Ruijsenaars operators, in the case

\footnotetext{
${ }^{1}$ Supported in part by NSF grant DMS-9501290.
} 
of integer coupling constant. For more general representations, one obtains some "spin generalizations" of Ruijsenaars operators.

The transfer matrix associated to the top exterior power turns out to be related to the quantum determinant, a difference operator which is central in the "operator algebra" associated to the elliptic quantum group.

In previous papers EV4, FV5, a dynamical version of the Bethe ansatz was developed, and the algebraic integrability of the Ruijsenaars model was proved in the $A_{1}$ case. It is likely that a dynamical version of the "nested" Bethe ansatz (a recursive Bethe ansatz for $A_{N-1}$ ) can be used to study the eigenvalue problem for these families of commuting operators.

Let us conclude this introduction by making some comments on related papers. The representation theory of elliptic quantum groups was described in FV3 for the $A_{1}$-case. In the trigonometric degeneration, the Ruijsenaars operators become the Macdonald difference operators. They are related to the representation theory of finite dimensional quantum groups, as pointed out by Etingof and Kirillov EK]. In fact, in this case, one can construct them as transfer matrices with dynamical $R$-matrices without spectral parameters, see ABB. In the elliptic case, Ruijsenaars operators can also be obtained as transfer matrices associated to the Sklyanin algebra, as shown by Hasegawa [H]. His construction appears to be related to ours, but is more complicated due to the complexity of the vertex-IRF transformation relating the two approaches. Hasegawa also gives a space of theta functions which is invariant under the action of the Ruijsenaars operators. Acknowledgement. We are grateful to Ivan Cherednik for inspiring discussions on the fusion procedure.

\section{The Elliptic QUANTUM GROUP ASSOCIATED TO $g l_{N}$}

We review here the definition of the elliptic quantum group $E=E_{\tau, \gamma / 2}\left(g l_{N}\right)$ associated to $g l_{N}[\mathbb{F}$ (or rather of its representations) and the construction of commuting transfer matrices associated to representations of $E$ [FV4].

Let $\mathfrak{h}$ be the Cartan subalgebra of $g l_{N}$. It is the Abelian Lie algebra of diagonal complex $N \times N$ matrices. We identify $\mathfrak{h}$ with its dual space via the nondegenerate bilinear form $(x, y)=\operatorname{tr}(x y)$, and with $\mathbb{C}^{N}$ via the orthonormal basis $\omega_{j}=$ $\operatorname{diag}(0, \ldots, 0,1,0, \ldots, 0)$ with a 1 in the $j$ th position $(j=1, \ldots, N)$.

A finite dimensional diagonalisable $\mathfrak{h}$-module is a complex finite dimensional vector space $W$ with a weight decomposition $W=\oplus_{\mu \in \mathfrak{h}^{*}} W[\mu]$, so that $\mathfrak{h}$ acts on $W[\mu]$ by $x w=\mu(x) w,(x \in \mathfrak{h}, w \in W[\mu])$. For example, the vector representation of $g l_{N}$ is $V=\mathbb{C}^{N}$ with standard basis $e_{1}, \ldots, e_{N}$ and with non-zero weight spaces $V\left[\omega_{j}\right]=\mathbb{C} e_{j}$, $j=1, \ldots, N$

Let us fix a point $\tau$ in the upper half plane and a generic complex number $\gamma$. Let

$$
\theta(z)=-\sum_{j \in \mathbb{Z}+\frac{1}{2}} e^{\pi i j^{2} \tau+2 \pi i j\left(z+\frac{1}{2}\right)}
$$

be Jacobi's first theta function. 
Let $R(z, \lambda) \in \operatorname{End}\left(\mathbb{C}^{N} \otimes \mathbb{C}^{N}\right)$ be the $R$-matrix of the elliptic quantum group $E=$ $E_{\tau, \gamma / 2}\left(g l_{N}\right)$. It is a function of the spectral parameter $z \in \mathbb{C}$ and an additional variable $\lambda=\left(\lambda_{1}, \ldots, \lambda_{N}\right) \in \mathfrak{h}^{*}$ in the dual of the Cartan subalgebra of $g l_{N}$. It is a solution of the dynamical Yang-Baxter equation

$$
\begin{aligned}
& R\left(z_{1}-z_{2}, \lambda-\gamma h^{(3)}\right)^{(12)} R\left(z_{1}-z_{3}, \lambda\right)^{(13)} R\left(z_{2}-z_{3}, \lambda-\gamma h^{(1)}\right)^{(23)} \\
& \quad=R\left(z_{2}-z_{3}, \lambda\right)^{(23)} R\left(z_{1}-z_{3}, \lambda-\gamma h^{(2)}\right)^{(13)} R\left(z_{1}-z_{2}, \lambda\right)^{(12)}
\end{aligned}
$$

and is "unitary":

$$
R(z, \lambda) R(-z, \lambda)^{(21)}=\operatorname{Id}_{\mathbb{C}^{N} \otimes \mathbb{C}^{N}}
$$

We adopt a standard notation: for instance, $R\left(z, \lambda-\gamma h^{(3)}\right)^{(12)}$ acts on a tensor $v_{1} \otimes v_{2} \otimes v_{3}$ as $R\left(z, \lambda-\gamma \mu_{3}\right) \otimes$ Id if $v_{3}$ has weight $\mu_{3}$. The formula for $R$ is

$$
R(z, \lambda)=\sum_{i=1}^{N} E_{i, i} \otimes E_{i, i}+\sum_{i \neq j} \alpha\left(z, \lambda_{i}-\lambda_{j}\right) E_{i, i} \otimes E_{j, j}+\sum_{i \neq j} \beta\left(z, \lambda_{i}-\lambda_{j}\right) E_{i, j} \otimes E_{j, i} .
$$

The functions $\alpha, \beta$ are ratios of theta functions:

$$
\alpha(z, \lambda)=\frac{\theta(z) \theta(\lambda+\gamma)}{\theta(z-\gamma) \theta(\lambda)}, \quad \beta(z, \lambda)=-\frac{\theta(z+\lambda) \theta(\gamma)}{\theta(z-\gamma) \theta(\lambda)}
$$

and $E_{i, j}$ is the matrix such that $E_{i, j} e_{k}=\delta_{j, k} e_{i}$. Note that $R(z, \lambda)$ is invariant under the symmetric group $S_{N}$ (the Weyl group of $g l_{N}$ ), in the sense that for any permutation $\sigma$

$$
R(z, \sigma \cdot \lambda)=\sigma \otimes \sigma R(z, \lambda) \sigma^{-1} \otimes \sigma^{-1},
$$

where $S_{N}$ acts linearly on $\mathfrak{h}$ and $\mathbb{C}^{N}$ by permutation of coordinates.

A representation of the elliptic quantum group $E$ (an $E$-module) is by definition a pair $(W, L)$ where $W$ is a (say finite-dimensional, diagonalisable) $\mathfrak{h}$-module and $L(z, \lambda)$ is a meromorphic function with values in $\operatorname{End}_{\mathfrak{h}}\left(\mathbb{C}^{N} \otimes W\right)$ (the endomorphisms commuting with the action of $\mathfrak{h}$ ), obeying the relations

$$
\begin{aligned}
& R\left(z_{1}-z_{2}, \lambda-\gamma h^{(3)}\right)^{(12)} L\left(z_{1}, \lambda\right)^{(13)} L\left(z_{2}, \lambda-\gamma h^{(1)}\right)^{(23)} \\
& \quad=L\left(z_{2}, \lambda\right)^{(23)} L\left(z_{1}, \lambda-\gamma h^{(2)}\right)^{(13)} R\left(z_{1}-z_{2}, \lambda\right)^{(12)} .
\end{aligned}
$$

An $E$-submodule of an $E$-module $(W, L)$ is a pair $\left(W_{1}, L_{1}\right)$ where $W_{1}$ is an $\mathfrak{h}$-submodule of $W$ such that $\mathbb{C}^{n} \otimes W_{1}$ is invariant under the action of all the $L(z, \lambda)$ and $L_{1}(z, \lambda)$ is the restriction to this invariant subspace. $E$-submodules are $E$-modules.

The basic example of an $E$-module is $\left(\mathbb{C}^{N}, L\right)$ with $L(z, \lambda)=R(z-w, \lambda)$. It is called the vector representation with evaluation point $w$ and is denoted by $V(w)$.

Other modules can be obtained by taking tensor products: if $\left(W_{1}, L_{1}\right)$ and $\left(W_{2}, L_{2}\right)$ are $E$-modules, then also $\left(W_{1} \otimes W_{2}, L\right)$, with $L(z, \lambda)=L_{1}\left(z, \lambda-\gamma h^{(3)}\right)^{(12)} L_{2}(z, \lambda)^{(13)}$.

The transfer matrix associated to an $E$-module $(W, L)$ is a difference operator acting on the space $F(W[0])$ of meromorphic functions of $\lambda \in \mathfrak{h}^{*}$ with values in the zero-weight 
space of $W$. It is defined by the formula

$$
T(z) f(\lambda)=\sum_{\mu} \operatorname{tr}_{V[\mu]}^{(1)} L(z, \lambda) f(\lambda-\gamma \mu) .
$$

The trace is over the (one-dimensional) weight spaces of $V=\mathbb{C}^{N}$. More explicitly, let us introduce matrix elements by $L(z, \lambda) e_{i} \otimes v=\sum_{j} e_{j} \otimes L_{j i}(z, \lambda) v$. Then

$$
T(z) f(\lambda)=\sum_{i=1}^{N} L_{i i}(z, \lambda) f\left(\lambda-\gamma \omega_{i}\right) .
$$

It follows from the Yang-Baxter equation that the transfer matrices commute for different values of the spectral parameters. For tensor products of vector representations one recovers in this way the transfer matrices of IRF models. In this paper we consider another class of modules.

\section{Symmetric AND EXTERIOR POWERS OF THE VECTOR REPRESENTATION}

For any $n=1,2, \ldots$, the symmetric group $S_{n}$ acts on $\left(\mathbb{C}^{N}\right)^{\otimes n}=\mathbb{C}^{N} \otimes \cdots \otimes \mathbb{C}^{N}$ by permuting the factors. A tensor in $\left(\mathbb{C}^{N}\right)^{\otimes n}$ is called symmetric if it is invariant under the symmetric group. We denote by $S^{n}\left(\mathbb{C}^{N}\right)$ the space of symmetric tensors. We denote by $\wedge^{n}\left(\mathbb{C}^{N}\right)$ the $n$th exterior power of $\mathbb{C}^{N}$. It is the quotient of $\left(\mathbb{C}^{N}\right)^{\otimes n}$ by the subspace $J_{n}\left(\mathbb{C}^{N}\right)$ spanned by the tensors of the form $\sigma v-\epsilon(\sigma) v, \sigma \in S_{n}, v \in\left(\mathbb{C}^{N}\right)^{n}$. Here $\epsilon(\sigma)$ is the sign of the permutation $\sigma$.

The $R$ matrix is invertible for generic values of the parameters. It becomes singular at special values of the spectral parameter. As is well-known in the non-dynamical case, these singularities are responsible for the reducibility of the generically irreducible tensor products of evaluation representations at special evaluation points.

Lemma 3.1. Let $\gamma$ and $\lambda \in \mathfrak{h}^{*}$ be generic. Then $R(z, \lambda)$ is a nonsingular matrix for all $z \neq \pm \gamma$ (modulo $\mathbb{Z}+\tau \mathbb{Z})$.

(i) The image of $R(-\gamma, \lambda)$ is $S^{2}\left(\mathbb{C}^{N}\right)$

(ii) The kernel of $R^{\mathrm{reg}}(\gamma, \lambda) \equiv \operatorname{res}_{z=\gamma} R(z, \lambda)$ is $J_{2}\left(\mathbb{C}^{N}\right)=S^{2}\left(\mathbb{C}^{N}\right)$

Proof: We have the "unitarity property" $R(z, \lambda) R(-z, \lambda)^{(21)}=1$ which implies that $R(z, \lambda)$ is nonsingular unless $z$ or $-z$ is a pole. This occurs only if $z= \pm \gamma$. The other claims follow easily from the definition of $R$.

Let us define operators $W_{n}(z, \lambda) \in \operatorname{End}\left(\left(\mathbb{C}^{N}\right)^{\otimes n}\right)\left(z \in \mathbb{C}^{n}, \lambda \in \mathfrak{h}^{*}\right)$ recursively by the conditions:

$$
\begin{aligned}
W_{1}(z, \lambda)= & 1 \\
W_{n+1}(z, \lambda)= & R\left(z_{1}-z_{2}, \lambda-\gamma \sum_{j=3}^{n+1} h^{(j)}\right)^{(12)} \cdots R\left(z_{1}-z_{n}, \lambda-\gamma h^{(n+1)}\right)^{(1 n)} \\
& R\left(z_{1}-z_{n+1}, \lambda\right)^{(1 n+1)}\left(1 \otimes W_{n}\left(z_{2}, \ldots, z_{n+1}, \lambda-\gamma h^{(1)}\right)\right) .
\end{aligned}
$$



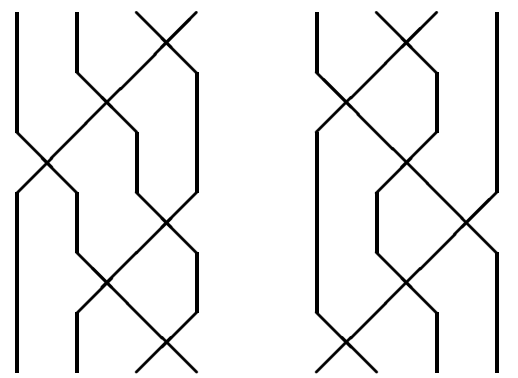

Figure 1. Two expressions for $W_{4}(z, \lambda)$

For instance, $W_{2}(z, \lambda)=R\left(z_{1}-z_{2}, \lambda\right)$ and $W_{3}(z, \lambda)$ is the left-hand side of the dynamical Yang-Baxter equation. It has therefore two expressions as a product of three $R$-matrices.

Similarly, $W_{n}$ can be written in several different ways as a product of $n(n-1) / 2 R$ matrices. This is most easily seen using a graphical representation. The left part of Fig. 11 represents the expression obtained by using the recursive definition. Let us number the lines of the drawing from 1 to $n$ from left to right at the bottom of the drawing. Every crossing between a line $j$ and a line $k$ represents an $R$-matrix

$$
R\left(z_{j}-z_{k}, \lambda-\gamma \sum h^{(l)}\right)^{(j k)},
$$

where we adopt the convention that the line numbered $j$ is at the left of the line $k$ below the crossing. The sum is over the numbers $l$ assigned to the lines at the left of the crossing. The expression represented by the drawing is then the product of these $R$-matrices, the ordering being determined by reading the drawing from the bottom to the top, so that the bottom crossing corresponds to the rightmost $R$-matrix. Using this rule, one can assign a product of $R$-matrices to any drawing obtained by putting vertical segments and crossings on top of each other, as in Fig. 1, so as to obtain $n$ lines connecting $n$ points at the bottom with $n$ points at the top. A drawing of this kind will be called a diagram. To each diagram $D$ with $n$ lines we thus associate a function $W_{D}(z, \lambda)$ on $\mathbb{C}^{n} \times \mathfrak{h}^{*}$ with values in the endomorphisms of $\left(\mathbb{C}^{N}\right)^{\otimes n}$ Every diagram with $n$ lines induces a permutation of $n$ letters: it sends $j$ to $k$ if the $j$ th point at the bottom is connected to the $k$ th point at the top.

Lemma 3.2. If two diagrams $D, D^{\prime}$ induce the same permutation then $W_{D}(z, \lambda)=$ $W_{D^{\prime}}(z, \lambda)$.

Proof: We use the fact that the symmetric group $S_{n}$ is generated by adjacent transpositions $s_{1}, \ldots, s_{n-1}$ with relations (a) $s_{j}^{2}=1$, (b) $s_{j} s_{j+1} s_{j}=s_{j+1} s_{j} s_{j+1}$, (c) $s_{j} s_{k}=s_{k} s_{j}$ if $|j-k| \geq 2$. To a diagram we associate in an obvious way a word in the generators $s_{j}$, so that its image in $S_{n}$ is the induced permutation. For example, we associate the word $s_{3} s_{2} s_{1} s_{3} s_{2} s_{3}$ to the diagram on the left in Fig. 1. If two diagrams induce the same permutation, the corresponding words can be obtained from each other by applying a sequence of relations. To each relation there corresponds a property of $R$-matrices that 
implies that the corresponding endomorphisms $W_{D}(z, \lambda)$ coincide: namely, we have (a) the "unitarity", (b) the dynamical Yang-Baxter equation, and (c) $R^{(j, k)}\left(z, \lambda-\sum_{l \in L} h^{(l)}\right)$ commutes with $R^{(r, s)}\left(z, \lambda-\sum_{l \in K} h^{(l)}\right)$ if the sets $\{j, k\}$ and $\{r, s\}$ are disjoint, and are either contained in or have empty intersection with $L$ and $K$.

Corollary 3.3. Let us call a diagram admissible if every line crosses every other line precisely once. The products of $R$-matrices associated to any admissible diagram are all equal to $W_{n}(z, \lambda)$.

Definition. Let $z^{S}=(0, \gamma, 2 \gamma, \ldots,(n-1) \gamma), z^{\wedge}=((n-1) \gamma, \ldots, 2 \gamma, \gamma, 0)$. We set

$$
\begin{aligned}
& W_{n}^{S}(\lambda)=W_{n}\left(z^{S}, \lambda\right) \\
& W_{n}^{\wedge}(\lambda)=\lim _{z \rightarrow z^{\wedge}} \prod_{j=1}^{N-1}\left(z_{j}-z_{j+1}-\gamma\right) W_{n}(z, \lambda)^{(n, \ldots, 1)} .
\end{aligned}
$$

Remark. Note that the spectral parameter in any of the $R$-matrices of the product defining $W_{n}^{S}(\lambda)$ is always a negative multiple of $\gamma$, so that there are no divergent $R$-matrices in this product. Similarly, $W_{n}^{\wedge}(\lambda)$ is a product of $R$-matrices with spectral parameter $k \gamma$, with $k=2,3, \ldots$, which are finite, and "regularized" $R$-matrices $R^{\mathrm{reg}}(\gamma, \lambda)=\operatorname{res}_{z=\gamma} R(z, \lambda)$.

Proposition 3.4. Let $\lambda$ be generic. Then

(i) The image of $W_{n}^{S}(\lambda)$ is equal to $S^{n}\left(\mathbb{C}^{N}\right)$.

(ii) The kernel of $W_{n}^{\wedge}(\lambda)$ is equal to $J_{n}\left(\mathbb{C}^{N}\right)$.

Proof: The proofs of the two parts are similar: one inclusion is a consequence of Lemma 3.1 and Corollary 3.3. The other inclusion is shown by counting dimensions at $\gamma=0$. We use the fact that the dimension of the image (kernel) of a holomorphic family of matrices at a generic point is at least (at most) the dimension at a special point.

Let $P$ denote the flip $u \otimes v \mapsto v \otimes u$ on $\mathbb{C}^{N} \otimes \mathbb{C}^{N}$. Since every permutation is a product of adjacent transpositions, we have

$$
\begin{aligned}
S^{n}\left(\mathbb{C}^{N}\right) & =\left\{v \in\left(\mathbb{C}^{N}\right)^{\otimes n} \mid P^{(j, j+1)} v=v, \quad j=1, \ldots, n-1\right\}, \\
J_{n}\left(\mathbb{C}^{N}\right) & =\sum_{j=1}^{N-1}\left\{P^{(j, j+1)} v+v \mid v \in\left(\mathbb{C}^{N}\right)^{\otimes n}\right\} .
\end{aligned}
$$

(i) Let us first show that the image of $W_{n}^{S}(\lambda)$ is contained in $S^{n}\left(\mathbb{C}^{N}\right)$. It suffices to show that $P^{(j, j+1)} W_{n}^{S}(\lambda)=W_{n}^{S}(\lambda)$ for all $j=1, \ldots, n-1$. This follows from Lemma 3.1 (i), and and the fact that we can always find an admissible diagram such that the highest crossing is the one between line $j$ and line $j+1$ (take any admissible diagram and "move" lines $j, j+1$ so as to push the crossing to the top).

Let us now show the converse, by considering the limit $\gamma \rightarrow 0$. The operator $W_{n}^{S}(\lambda)$ is a product of $R$-matrices whose spectral parameter is a negative integer multiple of 
$\gamma$. Let us write $R_{\gamma}(z, \lambda)$ for our $R$-matrix to make the $\gamma$-dependence apparent. The $R$-matrix at $z=-k \gamma(k=1,2, \ldots)$ is then regular as a function of $\gamma$ at $\gamma=0$ and its limit

$$
\lim _{\gamma \rightarrow 0} R_{\gamma}(-k \gamma, \lambda)=\frac{k}{k+1} \operatorname{Id}+\frac{1}{k+1} P,
$$

acts as the identity on $S^{2}\left(\mathbb{C}^{N}\right)$. Hence, if $\gamma=0, W_{n}^{S}(\lambda)$ acts on symmetric tensors as the identity and thus the image contains all symmetric tensors. It follows that, for generic $\gamma$, the dimension of the image is at least the dimension of $S^{n}\left(\mathbb{C}^{N}\right)$. But since the image is contained in $S^{n}\left(\mathbb{C}^{N}\right)$, it must coincide with it.

(ii) We first show that the kernel of $W_{n}^{\wedge}(\lambda)$ contains $J_{n}\left(\mathbb{C}^{N}\right)$. If a vector is of the form $w=P^{(j, j+1)} v+v$, then $W_{n}^{\wedge}(\lambda)$ vanishes on it, as follows from Lemma 3.1 using a representation of $W_{n}^{\wedge}(\lambda)$ corresponding to an admissible diagram such that the crossing between line $j$ and line $j+1$ is at the bottom.

Let us now show that, in the generic case, the kernel of $W_{n}^{\wedge}(\lambda)$ is contained in $J_{n}\left(\mathbb{C}^{N}\right)$. Let $A_{n}\left(\mathbb{C}^{N}\right)=\left\{v \in\left(\mathbb{C}^{N}\right)^{\otimes n} \mid P^{(j, j+1)} v=-v, \quad j=1, \ldots, n\right\}$ be the space of antisymmetric tensors. We have a direct sum decomposition $\left(\mathbb{C}^{N}\right)^{\otimes n}=J_{n}\left(\mathbb{C}^{N}\right) \oplus A_{n}\left(\mathbb{C}^{N}\right)$. Indeed, $A_{n}\left(\mathbb{C}^{N}\right)$ is the orthogonal complement of $J_{n}\left(\mathbb{C}^{N}\right)$ with respect to the product of standard inner products.

As $\gamma \rightarrow 0$, we have

$$
\begin{aligned}
\lim _{\gamma \rightarrow 0} R_{\gamma}(k \gamma, \lambda) & =\frac{k}{k-1} \mathrm{Id}-\frac{1}{k-1} P, \quad k=2,3, \ldots \\
\lim _{\gamma \rightarrow 0} \frac{1}{\gamma} R_{\gamma}^{\mathrm{reg}}(\gamma, \lambda) & =\mathrm{Id}-P .
\end{aligned}
$$

Thus, for $\gamma=0, \gamma^{-n+1} W_{n}^{\wedge}(\lambda)$ acts as a non-zero multiple of the identity on $A_{n}\left(\mathbb{C}^{N}\right)$. The dimension of the kernel is therefore at most the dimension of $J_{n}\left(\mathbb{C}^{N}\right)$, and the claim follows.

Theorem 3.5. Let, for $n=0,1,2, \ldots, V^{\otimes n}(w)$ denote the E-module $V(w) \otimes V(w+$ $\gamma) \otimes \cdots \otimes V(w+\gamma(n-1))$. Then

(i) The subspace $S^{n}\left(\mathbb{C}^{N}\right)$ is an E-submodule of $V^{\otimes n}(w)$.

(ii) The subspace $J_{n}\left(\mathbb{C}^{N}\right)$ is an E-submodule of $V^{\otimes n}(w)$.

Definition. The $E$-module $S^{n}\left(\mathbb{C}^{N}\right)$ of (i) is called the $n$th symmetric power of the vector representation with evaluation point $w$ and is denoted by $S^{n} V(w)$. The quotient $V^{\otimes n}(w) / J_{n}\left(\mathbb{C}^{N}\right)$ by the submodule $J_{n}\left(\mathbb{C}^{N}\right)$ of (ii) is called the $n$th exterior power of the vector representation with evaluation point $w$ and is denoted by $\wedge^{n} V(w)$.

What the theorem means is that the $L$-operator on $\mathbb{C}^{N} \otimes\left(\mathbb{C}^{N}\right)^{\otimes n}$

$$
\begin{aligned}
L(z, \lambda)= & R\left(z-w, \lambda-\gamma \sum_{j=2}^{n} h^{(j)}\right)^{(01)} R\left(z-w-\gamma, \lambda-\gamma \sum_{j=3}^{n} h^{(j)}\right)^{(02)} \\
& \cdots R(z-w-\gamma(n-1), \lambda)^{(0 n)}
\end{aligned}
$$


(the factors in the tensor products are numbered from 0 to $n$ ) preserves the subspaces $\mathbb{C}^{N} \otimes S^{n}\left(\mathbb{C}^{N}\right)$ and $\mathbb{C}^{N} \otimes J_{n}\left(\mathbb{C}^{N}\right)$.

In order to prove this theorem, we introduce another operator on $\mathbb{C}^{N} \otimes\left(\mathbb{C}^{N}\right)^{\otimes n}$. It is the $L$-operator corresponding to the "opposite coproduct":

$$
\begin{aligned}
L^{\prime}(z, \lambda)= & R\left(z-w-\gamma(n-1), \lambda-\gamma \sum_{j=1}^{n-1} h^{(j)}\right)^{(0 n)} \\
& \cdots R\left(z-w-\gamma, \lambda-\gamma h^{(1)}\right)^{(02)} R(z-w, \lambda)^{(01)} .
\end{aligned}
$$

\section{Lemma 3.6.}

(i) $L(z, \lambda)\left(1 \otimes W_{n}^{S}\left(\lambda-\gamma h^{(0)}\right)\right)=\left(1 \otimes W_{n}^{S}(\lambda)\right) L^{\prime}(z, \lambda)$.

(ii) $\left(1 \otimes W_{n}^{\wedge}(\lambda)\right) L(z, \lambda)=L^{\prime}(z, \lambda)\left(1 \otimes W_{n}^{\wedge}\left(\lambda-\gamma h^{(0)}\right)\right)$.

Proof: (i) The left-hand side is, by definition, $W_{n+1}(Z, \lambda)$, with

$$
Z=(z, w, w+\gamma, \ldots, w+(n-1) \gamma),
$$

with the notational convention that the factors are numbered from 0 to $n$. The right-hand side is another representation of $W_{n+1}(Z, \lambda)$ as a product of $R$-matrices. It corresponds to the diagram on the right in Fig. 1. The proof of (ii) is similar: the claim follows from the identity between two representations of $W_{n+1}(Z, \lambda)^{(n, \ldots, 1)}$ in the limit

$$
Z \rightarrow(z, w+(n-1) \gamma, \ldots, 2 \gamma, \gamma)
$$

Theorem 3.5 follows from this lemma and Prop. 3.4.

Example. The top exterior power $\wedge^{N} V(w)$ is a one-dimensional E-module. The matrix elements of the $L$-operator are

$$
L_{i j}(z, \lambda)=\delta_{i j} \frac{\theta(z-w-\gamma)}{\theta(z-w-\gamma N)} \prod_{k: k \neq i} \frac{\theta\left(\lambda_{i}-\lambda_{k}-\gamma\right)}{\theta\left(\lambda_{i}-\lambda_{k}\right)}
$$

\section{RuijsenAars operators}

The Ruijsenaars operators $[\mathrm{R}]$ are integrable difference operators that give a $q$-deformation of the Calogero-Moser integrable differential operators. They act on functions on $\mathfrak{h}^{*} \simeq \mathbb{C}^{N}$ Let $\Gamma_{j}$ be the shift by $-\gamma \omega_{j}$ :

$$
\Gamma_{j} f\left(\lambda_{1}, \ldots, \lambda_{N}\right)=f\left(\lambda_{1}, \ldots, \lambda_{j}-\gamma, \ldots, \lambda_{N}\right),
$$

and let $\ell$ be a non-negative integer (the "coupling constant"). The corresponding Ruijsenaars operator is (up to conjugation by a function, see [H])

$$
M=\sum_{i=1}^{N} \prod_{j: j \neq i} \frac{\theta\left(\lambda_{i}-\lambda_{j}+\ell \gamma\right)}{\theta\left(\lambda_{i}-\lambda_{j}\right)} \Gamma_{i}
$$


It is a symmetric difference operator: if $\sigma \in S_{N}$ acts on functions by $\sigma f(\lambda)=f\left(\sigma^{-1} \lambda\right)$, then $\sigma M=M \sigma$ for all $\sigma \in S_{N}$.

Let us consider the transfer matrix associated to the symmetric power $S^{n} V(0)$. The zero weight space of this module is trivial unless $n$ is a multiple of $N$. If $n=N \ell$ then the zero weight space is one-dimensional and is spanned by the sum of the tensors $e_{i_{1}} \otimes \cdots \otimes e_{i_{N \ell}}$ over all sequences $\left(i_{j}\right)$ such that each number between 1 and $N$ occurs precisely $\ell$ times. Let us denote this sum by $e$.

Theorem 4.1. Let us identify $S^{N \ell}\left(\mathbb{C}^{N}\right)[0]$ with $\mathbb{C}$ using the basis e, and let $T(z)$ be the transfer matrix associated to $S^{N \ell} V(0)$. Then

$$
T(z)=\frac{\theta(z-\gamma \ell)}{\theta(z-\gamma N \ell)} M
$$

The rest of this section contains the proof of this theorem.

Lemma 4.2. Let $\bar{e}$ be the weight zero tensor in $\left(\mathbb{C}^{N}\right)^{\otimes N \ell}$,

$$
\bar{e}=e_{1} \otimes \cdots \otimes e_{1} \otimes \cdots \otimes e_{N} \otimes \cdots \otimes e_{N},
$$

where each $e_{j}$ appears $\ell$ times. Let

$$
g_{N, \ell}(\lambda)=\prod_{1 \leq j<k \leq N} \prod_{s=1}^{\ell} \frac{\theta\left(\lambda_{j}-\lambda_{k}+\gamma s\right)}{\theta\left(\lambda_{j}-\lambda_{k}-\gamma(s-1)\right)} .
$$

Then there exists a non-zero constant $C_{N, \ell}$ such that

$$
W_{N \ell}^{S}(\lambda) \bar{e}=C_{N, \ell} g_{N, \ell}(\lambda) e .
$$

Proof: We know from Prop. 3.4 that the left-hand side must be proportional to the symmetric tensor $e$. It suffices therefore to compute the coefficient of $\bar{e}$ in this expression. It is easy to see that only diagonal elements in the $R$-matrices give a non-trivial contribution to this coefficient. Therefore $g_{N, \ell}$ is just a product of functions

$$
\alpha(z, \lambda)=\frac{\theta(z) \theta(\lambda+\gamma)}{\theta(z-\gamma) \theta(\lambda)}
$$

The values of $z$ occurring here are of the form $m \gamma$ for some negative integer $m$, so that no zeros or divergencies appear. The $z$-dependent factors contribute to $C_{N, \ell}$.

Let us compute the $\lambda$-dependent part of the product of functions $\alpha$, using a representation of $W_{N \ell}^{S}$ by a diagram. As above, we number the lines from 1 to $N \ell$ from left to right at the bottom of the diagram. Let us say that the first $\ell$ lines have weight 1 , the next $\ell$ lines have weight 2 and so on. Let us compute the contribution to the product of a crossing between a line of weight $j$ and a line of weight $k \neq j$ : suppose that there are $n_{i}$ lines of weight $i$ to the left of the crossing. Then the crossing contributes $\alpha\left(z, \lambda_{j}-\lambda_{k}-\gamma\left(n_{j}-n_{k}\right)\right.$ ) (for some $z$ ) to the product. Crossings between lines with the same weight give a trivial contribution to the product. 
Taking together all crossings between lines of weight $j$ and $k$, and then taking the product over all $j<k$ yields

$$
g_{N, \ell}(\lambda)=C_{N, \ell} \prod_{j<k} \prod_{r, s=1}^{\ell-1} \frac{\theta\left(\lambda_{j}-\lambda_{k}-\gamma(r-s)+\gamma\right)}{\theta\left(\lambda_{j}-\lambda_{k}-\gamma(r-s)\right)}
$$

which can be simplified to the desired expression.

We can now complete the proof of the theorem. For any scalar function $f(\lambda)$,

$$
\begin{aligned}
T(z) f(\lambda) e & =\sum_{j} \operatorname{tr}_{V\left[\omega_{j}\right]}^{(0)} L(z, \lambda) e \Gamma_{j} f(\lambda) \\
& =\sum_{j} \operatorname{tr}_{V\left[\omega_{j}\right]}^{(0)} L(z, \lambda) C_{N, \ell}^{-1} g_{N, \ell}\left(\lambda-\omega_{j}\right)^{-1} W_{N \ell}^{S}\left(\lambda-\gamma \omega_{j}\right) \bar{e} \Gamma_{j} f(\lambda) .
\end{aligned}
$$

Since both sides of (3) are symmetric difference operators $(T(z)$ is symmetric as a consequence of (1)) with shifts by $-\omega_{1}, \ldots,-\omega_{N}$, it is sufficient to show that the coefficients of $\Gamma_{1}$ on both sides coincide. By Lemma 3.6,

$$
\begin{aligned}
\operatorname{tr}_{V\left[\omega_{1}\right]}^{(0)} L(z, \lambda) W_{N \ell}^{S}\left(\lambda-\gamma \omega_{1}\right) \bar{e} & =W_{N \ell}^{S}(\lambda) \operatorname{tr}_{V\left[\omega_{1}\right]}^{(0)} \hat{L}(z, \lambda) \bar{e} \\
& =W_{N \ell}^{S}(\lambda) \bar{e} \frac{\theta(z-\gamma \ell)}{\theta(z-\gamma N \ell)} \prod_{k=2}^{N} \frac{\theta\left(\lambda_{1}-\lambda_{k}\right)}{\theta\left(\lambda_{1}-\lambda_{k}-\gamma \ell\right)} \\
& =C_{N, \ell} g_{N, \ell}(\lambda) e \frac{\theta(z-\gamma \ell)}{\theta(z-\gamma N \ell)} \prod_{k=2}^{N} \frac{\theta\left(\lambda_{1}-\lambda_{k}\right)}{\theta\left(\lambda_{1}-\lambda_{k}-\gamma \ell\right)} .
\end{aligned}
$$

The second equality is obtained by using the explicit expression for the $R$-matrices. The calculation is simple, since only the diagonal entries give a non-vanishing contribution to the trace, and the product of $R$-matrices gives a product of functions $\alpha$ which factorize neatly, as in the proof of Lemma 4.2 .

It follows that the coefficient of $\Gamma_{1}$ in $T(z)$ is

$$
\frac{\theta(z-\gamma \ell)}{\theta(z-\gamma N \ell)} \prod_{k=2}^{N} \frac{\theta\left(\lambda_{1}-\lambda_{k}\right)}{\theta\left(\lambda_{1}-\lambda_{k}-\gamma \ell\right)} \frac{g_{\ell, N}(\lambda)}{g_{\ell, N}\left(\lambda-\gamma \omega_{1}\right)}=\frac{\theta(z-\gamma \ell)}{\theta(z-\gamma N \ell)} \prod_{k=2}^{N} \frac{\theta\left(\lambda_{1}-\lambda_{k}+\gamma \ell\right)}{\theta\left(\lambda_{1}-\lambda_{k}\right)} .
$$

We have used here the identity

$$
\frac{g_{\ell, N}(\lambda)}{g_{\ell, N}\left(\lambda-\gamma \omega_{1}\right)}=\prod_{k=2}^{N} \frac{\theta\left(\lambda_{1}-\lambda_{k}+\gamma \ell\right) \theta\left(\lambda_{1}-\lambda_{k}-\gamma \ell\right)}{\theta\left(\lambda_{1}-\lambda_{k}\right)^{2}} .
$$

\section{R-MATRICES, Higher RUIJSEnAARs OpERATORS AND THE DETERMinANT}

In the preceding section we have considered the $L$-operator $L(z, \lambda)$ of $S^{n} V(w)$, the $n$th symmetric powers of the vector representation, and its transfer matrices. In terms

of $R$-matrices (see the Appendix), $L(z, \lambda)$ is the $R$-matrix for the $E$-modules $V(z)$ and 
$S^{n} V(w)$. More generally, we may consider the $R$-matrices for $\wedge^{m} V(z)$ and $S^{n} V(w)$. The corresponding transfer matrices give then a family of commuting difference operators.

Let $V^{\otimes n}(z)=V(z) \otimes V(z+\gamma) \otimes \cdots \otimes V(z+(n-1) \gamma)$. Then we have $R$-matrices $\mathcal{R}_{V^{\otimes m}(z), V^{\otimes n}(w)}(\lambda)$ for $V^{\otimes m}(z)$ and $V^{\otimes n}(w)$ (if $z, w$ are generic) obeying the dynamical Yang-Baxter equation. They are products of fundamental $R$-matrices $R(z, \lambda)$, (cf. the Appendix) associated to a diagram where each of the first $m$ lines crosses each of the last $n$ lines precisely once.

Proposition 5.1. The R-matrix $\mathcal{R}_{V^{\otimes m}(z), V^{\otimes n}(w)}(\lambda)$ is invertible for generic $z, w$ and preserves the subspaces $A \otimes B$ where $A$ is $\left(\mathbb{C}^{N}\right)^{\otimes m}, S^{m}\left(\mathbb{C}^{N}\right)$ or $J_{m}\left(\mathbb{C}^{N}\right)$ and $B$ is $\left(\mathbb{C}^{N}\right)^{\otimes n}$, $S^{n}\left(\mathbb{C}^{N}\right)$ or $J_{n}\left(\mathbb{C}^{N}\right)$.

Proof: The invertibility follows from the fact that the product defining the $R$-matrix contains fundamental $R$-matrices whose spectral parameter is of the form $z-w+r \gamma$, with integer $r$, which are invertible for generic $z-w$. The other claim is proved by commuting the $R$-matrix with $W_{m}^{S, \wedge}(\lambda) \otimes \operatorname{Id}$ and $\operatorname{Id} \otimes W_{n}^{S, \wedge}(\lambda)$ using Lemma 3.2 as in the proof of Lemma 3.6. The details are left to the reader.

In particular we have invertible $R$-matrices $\mathcal{R}_{\wedge^{m} V(z), S^{n} V(w)}, \mathcal{R}_{\wedge^{m} V(z), \wedge^{n} V(w)}$ obeying dynamical Yang-Baxter equations for submodules and quotients. It follows that, if we set $\Gamma_{\mu} f(\lambda)=f(\lambda-\gamma \mu)$ (so that $\left.\Gamma_{j}=\Gamma_{\omega_{j}}\right)$ then the transfer matrices

$$
T_{m}(z)=\sum_{\mu} \operatorname{tr}_{\wedge^{m} V(z)[\mu]} \mathcal{R}_{\wedge^{m} V(z), S^{N \ell} V(0)} \Gamma_{\mu}, \quad m=1, \ldots N,
$$

are commuting difference operators on the space of functions of $\lambda \in \mathfrak{h}^{*}$ with values in $S^{N \ell} V(0)[0] \simeq \mathbb{C}$.

Here is an explicit formula for $T_{m}(z)$ in terms of the matrix elements of the $L$-operator of $S^{n} V(0)$ : let $|J|$ denote the cardinality of a subset $J$ of $\{1, \ldots, N\}$. Then

$$
\begin{gathered}
T_{m}(z) f(\lambda)=\sum_{1 \leq j_{1}<\cdots<j_{m} \leq N} \sum_{\sigma \in S_{m}} \epsilon(\sigma) L_{j_{\sigma(1)} j_{1}}\left(z, \lambda-\gamma\left(\omega_{j_{2}}+\cdots+\omega_{j_{m}}\right)\right) \\
\cdots L_{j_{\sigma(m)} j_{m}}(z-(m-1) \gamma, \lambda) f\left(\lambda-\gamma\left(\omega_{j_{1}}+\cdots+\omega_{j_{m}}\right)\right) .
\end{gathered}
$$

Let us compare these operators commuting with $M$ with the higher Ruijsenaars operators.

The Ruijsenaars operator $M$ is part of an algebra of $S_{N}$-symmetric commuting difference operators generated by $M_{1}=M, M_{2}, \ldots, M_{N}$, with

$$
M_{m}=\sum_{J,|J|=m} \prod_{j \in J, k \neq J} \frac{\theta\left(\lambda_{j}-\lambda_{k}+\gamma \ell\right)}{\theta\left(\lambda_{j}-\lambda_{k}\right)} \prod_{j \in J} \Gamma_{j} .
$$

Theorem 5.2. There exist non-zero meromorphic scalar functions $g_{m}(z, \gamma)$ so that

$$
T_{m}(z)=g_{m}(z, \gamma) M_{m}, \quad m=1, \ldots, N .
$$


Proof: The exterior powers of the vector representation have non-zero weight spaces $\wedge^{m}\left(\mathbb{C}^{N}\right)[\mu]=\mathbb{C} e_{j_{1}} \wedge \cdots \wedge e_{j_{m}}$, of weight $\mu=\sum_{i} \omega_{j_{i}}\left(j_{1}<\cdots<j_{m}\right)$. It follows that both sides of the equation we have to prove are $S_{N}$-symmetric difference operators of the form $\sum_{J,|J|=m} A_{J}^{m}(z, \lambda) \prod_{j \in J} \Gamma_{j}$. We also know that $T_{m}(z)$ commutes with $M$. This implies that the coefficients $A_{J}^{m}$ obey difference equations in $\lambda$. The idea it to show that these difference equations uniquely determine the coefficients up to multiplication by a constant.

The coefficients $A_{J}^{m}(z, \lambda)$ of $T_{m}(z)$ are meromorphic functions. As functions of $\lambda$ they are periodic in each $\lambda_{k}$ with period one and they depend only on the differences $\lambda_{j}-\lambda_{k}$. They can be thus considered as functions of $N-1$ of the variables $\lambda_{j}$.

Let $h(x)=\theta(x+\gamma) / \theta(x)$. If $j \in J$, the fact that $T_{m}(z)$ commutes with $M$ implies the difference equations

$$
A_{J}^{m}\left(z, \lambda-\gamma \omega_{j}\right)=\prod_{k \notin J} \frac{h\left(\lambda_{j}-\lambda_{k}-\gamma\right)}{h\left(\lambda_{j}-\lambda_{k}\right)} A_{J}^{m}(z, \lambda), \quad j \in J .
$$

It is easy to check that the coefficients appearing in $M_{m}$ also obey these difference equations. If $|J|=N$ or $N-1$, this is sufficient to prove the claim. Namely, in these cases we have a first order difference equation for all coefficients and all variables $\lambda_{j}$ (if $|J|=N-1$ we view $A_{J}^{m}$ as a function of $N-1$ variables $\left.\lambda_{j}, j \in J\right)$. We conclude that, for any $J$ with $|J| \geq N-1$, the ratio between the coefficient of $\prod_{j \in J} \Gamma_{j}$ in $T_{m}(z)$ and the coefficient of $\prod_{j \in J} \Gamma_{j}$ in $M_{m}$ is a meromorphic function $g_{J}(z, \lambda ; \gamma)$ which, as a function of $\lambda_{k}(1 \leq k \leq N)$, is periodic with period $\gamma$ and with period 1 . Taking $\gamma$ to be an irrational real number, we see that $g_{J}$ must be independent of $\lambda$. Since both $T_{m}(z)$ and $M_{m}$ are $S_{N}$-symmetric, all $g_{J}$ with $|J|=m$ must be equal to the same function $g_{m}$.

We have shown that $T_{m}(z)=g_{m}(z, \gamma) M_{m}$ for $m=N, N-1$ and some functions $g_{m}$ which are not identically zero since they do not vanish at $\gamma=0$, see below. In particular, this implies that $T_{m}(z)$ commutes with

$$
M_{N-1} M_{N}^{-1}=\sum_{j=1}^{N} \prod_{k \neq j} h\left(\lambda_{k}-\lambda_{j}\right) \Gamma_{j}^{-1}
$$

since $M_{N}=\prod_{j=1}^{N} \Gamma_{j}$. It follows that, for all $m$, the coefficients $A_{J}^{m}$ also obeys the difference equations with respect to the variables $\lambda_{j}, j \notin J$ :

$$
A_{J}^{m}\left(z, \lambda+\gamma \omega_{j}\right)=\prod_{k \in J} \frac{h\left(\lambda_{k}-\lambda_{j}-\gamma\right)}{h\left(\lambda_{k}-\lambda_{j}\right)} A_{J}^{m}(z, \lambda), \quad j \notin J
$$

of which the coefficients appearing in $M_{m}$ are clearly also a solution.

Proceeding as in the case $m=N, N-1$, we see that the difference equations uniquely determine all coefficients up to a factor independent of $\lambda$. By the $S_{N}$ symmetry, all factors (for fixed $m$ ) coincide. 
We have still to prove that the functions $g_{m}(z, \gamma)$ are not identically zero. As $\gamma \rightarrow 0$, $R(z, \lambda ; \gamma)$ tends to the identity, so $T_{m}(z)$ tends to $\sum_{J,|J|=m} \prod_{j \in J} \Gamma_{j}$. The same holds for $M_{m}$. It follows that $g_{m}(z, 0)=1$.

More generally, for any $E$-module $W$ with $L$-operator $L(z, \lambda)$, we have a family $T_{m}(z)$, $z \in \mathbb{C}, 1 \leq m \leq N$ of commuting difference operators on $W[0]$-valued functions, given by (田). In general the dimension of $W[0]$ is not one, so one gets vector valued generalizations of the Ruijsenaars model.

These operators are in the "operator algebra" FV1 of difference operators on $W$ valued functions of $\lambda$. By definition, the operator algebra is generated by the difference operators $\hat{L}_{i j}(z)=L_{i j}(z, \lambda) \Gamma_{j}, 1 \leq i, j, \leq N$. It is clear from (4) that the $T_{m}(z)$ are polynomials in these difference operators.

The transfer matrix $T_{N}(z)$ associated to the top exterior power is related to the quantum determinant (cf. [EV3], Section 10):

$$
T_{N}(z)=\frac{\phi(\lambda-\gamma h)}{\phi(\lambda)} \operatorname{Det}(z, \lambda) \prod_{j=1}^{N} \Gamma_{j}, \quad \phi(\lambda)=\prod_{i<j} \theta\left(\lambda_{i}-\lambda_{j}\right) .
$$

The difference operator $\widehat{\operatorname{Det}}(z)=\operatorname{Det}(z, \lambda) \prod \Gamma_{j}$ defined by this formula is a central element (for all $z$ ) of the operator algebra. This can be seen by writing the Yang-Baxter equation on $V(z) \otimes \wedge^{n} V(w) \otimes W$, using the formula in the example at the end of Section 3 for the $R$-matrix of the first two factors.

\section{Conclusions}

We have constructed some finite-dimensional modules over the elliptic quantum groups associated to $g l_{N}$. They are elliptic deformations of symmetric and exterior powers of the vector representation. Modules corresponding to more general Young diagrams will be considered elsewhere.

Transfer matrices associated to modules over elliptic quantum groups are commuting difference operators acting on functions with values in the zero weight space of the quantum space. We have considered here the special case where the zero weight space is one-dimensional.

In this case the commuting difference operators turn out to be essentially the Ruijsenaars operators. The advantage of this reformulation in terms of transfer matrices lies in the fact that we can apply the Bethe ansatz method to find eigenvectors. In the $g l_{2}$ case this was done in FV4, FV5]. In general, an adaptation of the "nested" Bethe ansatz to the dynamical case should give the result. In particular, one would get a quantum version of the spectral varieties of [FV1, FV2].

\section{Appendix A. $R$-MATRICES AND TRANSFER MATRICES}

We give here some details on $R$-matrices and commuting transfer matrices. In particular, we explain the interpretation of $R$-matrices as intertwiners for elliptic quantum 
groups and give the construction of commuting transfer matrices from $R$-matrices. The constructions are standard in quantum integrable systems based on the (non-dynamical) Yang-Baxter equation. All claims below follow easily from the definitions and from the dynamical Yang-Baxter equation.

Let $P_{V, W} \in \operatorname{Hom}(V \otimes W, W \otimes V)$ be the flip $v \otimes w \mapsto w \otimes v$. Let $W_{1}, W_{2}$ be $E$ modules with $L$-operators $L_{1}$ and $L_{2}$. A morphism (or intertwiner) from $W_{1}$ to $W_{2}$ is a meromorphic function $\phi: \mathfrak{h}^{*} \rightarrow \operatorname{Hom}_{\mathfrak{h}}\left(W_{1}, W_{2}\right)$ such that $\operatorname{Id} \otimes \phi(\lambda) L_{1}(z, \lambda)=$ $L_{2}(z, \lambda) \operatorname{Id} \otimes \phi\left(\lambda-\gamma h^{(1)}\right)$. An $R$-matrix for $W_{1}$ and $W_{2}$ is a meromorphic function $\mathcal{R}: \mathfrak{h}^{*} \rightarrow \operatorname{End}_{\mathfrak{h}}\left(W_{1} \otimes W_{2}\right)$ so that

$$
\mathcal{R}(\lambda) P_{W_{2}, W_{1}}: W_{2} \otimes W_{1} \rightarrow W_{1} \otimes W_{2},
$$

is a morphism.

For example $\mathcal{R}(\lambda)=R\left(z_{1}-z_{2}, \lambda\right)$ is an $R$-matrix for the evaluation modules $V\left(z_{1}\right)$ and $V\left(z_{2}\right)$, as a consequence of the dynamical Yang-Baxter equation. Let us call it the fundamental $R$-matrix with spectral parameter $z_{1}-z_{2}$.

Suppose that $W_{1}, W_{2}$ and $W_{3}$ are $E$-module and that $\mathcal{R}_{W_{i}, W_{j}}$ is an $R$-matrix for $W_{i}$ and $W_{j}(1 \leq i<j \leq 3)$. Then

$$
\begin{aligned}
& \mathcal{R}_{W_{1} \otimes W_{2}, W_{3}}(\lambda)=\mathcal{R}_{W_{1}, W_{3}}(\lambda)^{(13)} \mathcal{R}_{W_{2}, W_{3}}\left(\lambda-\gamma h^{(1)}\right)^{(23)} \\
& \mathcal{R}_{W_{1}, W_{2} \otimes W_{3}}(\lambda)=\mathcal{R}_{W_{1}, W_{3}}\left(\lambda-\gamma h^{(2)}\right)^{(13)} \mathcal{R}_{W_{1}, W_{2}}(\lambda)^{(12)}
\end{aligned}
$$

are $R$-matrices for $W_{1} \otimes W_{2}, W_{3}$ and for $W_{1}, W_{2} \otimes W_{3}$, respectively.

In particular, we see by iterating this construction that there are $R$-matrices $\mathcal{R}_{W_{1}, W_{2}}$, obtained as products of fundamental $R$-matrices, if $W_{1}, W_{2}$ are tensor products of vector representations with generic evaluation points. We have, for instance, $\mathcal{R}_{V(z), W}(\lambda)=$ $L_{W}(z, \lambda)$, the $L$ operator of the tensor product $W$ of vector representations. By construction, these $R$-matrices obey the dynamical Yang-Baxter equation

$$
\begin{gathered}
\mathcal{R}_{W_{1}, W_{2}}\left(\lambda-\gamma h^{(3)}\right)^{(12)} \mathcal{R}_{W_{1}, W_{3}}(\lambda)^{(13)} \mathcal{R}_{W_{2}, W_{3}}\left(\lambda-\gamma h^{(1)}\right)^{(23)} \\
=\mathcal{R}_{W_{2}, W_{3}}(\lambda)^{(23)} \mathcal{R}_{W_{1}, W_{3}}\left(\lambda-\gamma h^{(2)}\right)^{(13)} \mathcal{R}_{W_{1}, W_{2}}(\lambda)^{(12)}
\end{gathered}
$$

and the "unitarity" property

$$
\mathcal{R}_{W_{1}, W_{2}}(\lambda)^{(12)} \mathcal{R}_{W_{2}, W_{1}}(\lambda)^{(21)}=\operatorname{Id}_{W_{1} \otimes W_{2}} .
$$

In particular $R$-matrices for generic tensor products of vector representations are invertible.

Suppose that $\mathcal{R}_{W_{1}, W_{2}}$ is an $R$-matrix for $E$-modules $W_{1}, W_{2}$. The transfer matrix with "auxiliary space" $W_{1}$ and "quantum space" $W_{2}$ is the difference operator acting on functions on $\mathfrak{h}^{*}$ with values in the zero weight space $W_{2}[0]$ of $W_{2}$

$$
T_{W_{1}, W_{2}} f(\lambda)=\sum_{\mu \in \mathfrak{h}^{*}} \operatorname{tr}_{W_{1}[\mu]}\left(\mathcal{R}_{W_{1}, W_{2}}(\lambda)\right) f(\lambda-\gamma \mu)
$$


Proposition A.1. Suppose that $W_{1}, W_{2}, W_{3}$ are E-modules with $R$-matrices $\mathcal{R}_{W_{i}, W_{j}}$ obeying the dynamical Yang-Baxter equation. Let for $i=1,2 T_{i}=T_{W_{i}, W_{3}}$ be the transfer matrices with quantum space $W_{3}$ and assume that $\mathcal{R}_{W_{1}, W_{2}}(\lambda)$ is invertible for generic $\lambda$. Then $T_{1} T_{2}=T_{2} T_{1}$ on $W_{3}[0]$.

Proof: On $W_{1} \otimes W_{2} \otimes\left(W_{3}[0]\right)$ we can write the Yang-Baxter equation in the form

$$
\begin{aligned}
& \mathcal{R}_{W_{1}, W_{3}}(\lambda)^{(13)} \mathcal{R}_{W_{2}, W_{3}}\left(\lambda-\gamma h^{(1)}\right)^{(23)} \\
& \quad=\left(\mathcal{R}_{W_{1}, W_{2}}(\lambda)^{(12)}\right)^{-1} \mathcal{R}_{W_{2}, W_{3}}(\lambda)^{(23)} \mathcal{R}_{W_{1}, W_{3}}\left(\lambda-\gamma h^{(2)}\right)^{(13)} \mathcal{R}_{W_{1}, W_{2}}(\lambda)^{(12)} .
\end{aligned}
$$

Taking the trace over $W_{1} \otimes W_{2}$ yields the result.

\section{REFERENCES}

[ABB] J. Avan, O. Babelon and E. Billey, The Gervais-Neveu-Felder equation and the quantum Calogero-Moser systems Commun. Math. Phys. 178 (1996), 281-299

[C] I. Cherednik, Elliptic curves and soliton matrix differential equations, J. Sov. Math. 38 (1987), 1989-2027

[EK] P. I. Etingof and A. A. Kirillov, Jr., Macdonald's polynomials and representations of quantum groups, Math. Res. Lett. 1 (1994), 279-296

[F] G. Felder, Conformal field theory and integrable systems associated to elliptic curves, Proceedings of the International Congress of Mathematicians, Zürich 1994, p. 1247-1255, Birkhäuser, 1994; Elliptic quantum groups, Proceedings of the International Congress of Mathematical Physics, Paris 1994, 211-218, International Press 1995

[FV1] G. Felder and A. Varchenko, Integral representation of solutions of the elliptic KnizhnikZamolodchikov-Bernard equation, Int. Math. Res. Notices, No. 5(1995), 221-233

[FV2] G. Felder and A. Varchenko, Three formulae for eigenfunctions of integrable Schrödinger operators, hep-th/9511120, to appear in Comp. Math.

[FV3] G. Felder and A. Varchenko, On representations of the elliptic quantum group $E_{\tau, \eta}\left(s l_{2}\right)$ Commun. Math. Phys. 181 (1996), 741-761

[FV4] G. Felder and A. Varchenko, Algebraic Bethe ansatz for the elliptic quantum group $E_{\tau, \eta}\left(s l_{2}\right)$, Nucl. Phys. B480 (1996), 485-503

[FV5] G. Felder and A. Varchenko, Algebraic integrability of the two-body Ruijsenaars operator, $\mathrm{q}-$ alg/9610024

$[\mathrm{H}] \quad$ K. Hasegawa, Ruijsenaars' commuting difference operators as commuting transfer matrices, q-alg/9512029

[JKMO] M. Jimbo, A. Kuniba, T. Miwa and M. Okado, The $A_{n}^{(1)}$ face models, Commun. Math. Phys. 119 (1988), 543-565

[KRS] P. P. Kulish, N. Yu. Reshetikhin and E. K. Sklyanin, Yang-Baxter equation and representation theory I, Lett. Math. Phys. 5 (1981), 393-403

[R] S. N. M. Ruijsenaars, Complete integrability of relativistic Calogero-Moser systems and elliptic function identities, Commun. Math. Phys. 110 (1987), 191-213 